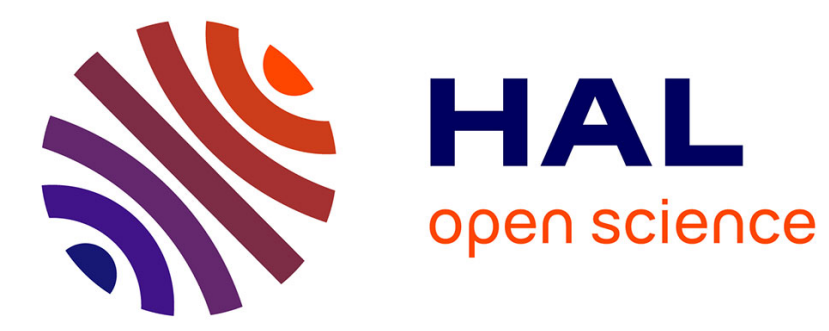

\title{
Free-space cavity optomechanics in a cryogenic environment
}

\author{
A. G. Kuhn, J. Teissier, L. Neuhaus, S. Zerkani, E. van Brackel, S. Deléglise, \\ T. Briant, P.-F. Cohadon, A. Heidmann, C. Michel, et al.
}

\section{- To cite this version:}

A. G. Kuhn, J. Teissier, L. Neuhaus, S. Zerkani, E. van Brackel, et al.. Free-space cavity optomechanics in a cryogenic environment. Applied Physics Letters, 2014, 104 (4), 10.1063/1.4863666 . in2p301357440

\section{HAL Id: in2p3-01357440 \\ https://hal.in2p3.fr/in2p3-01357440}

Submitted on 29 Aug 2016

HAL is a multi-disciplinary open access archive for the deposit and dissemination of scientific research documents, whether they are published or not. The documents may come from teaching and research institutions in France or abroad, or from public or private research centers.
L'archive ouverte pluridisciplinaire HAL, est destinée au dépôt et à la diffusion de documents scientifiques de niveau recherche, publiés ou non, émanant des établissements d'enseignement et de recherche français ou étrangers, des laboratoires publics ou privés. 


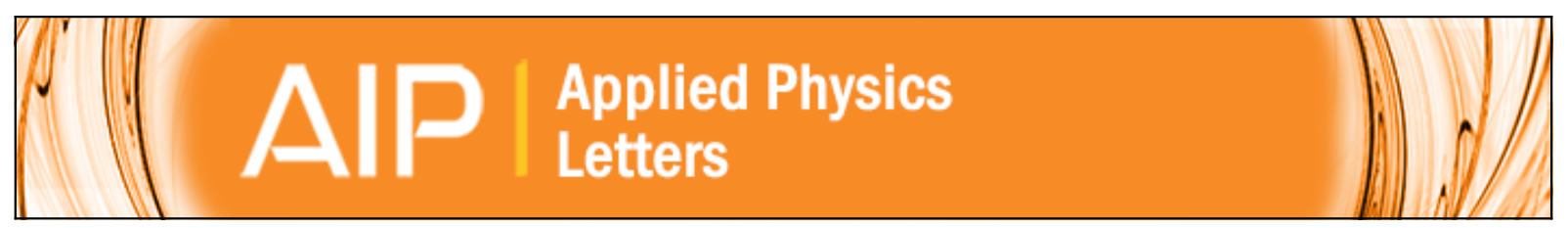

\section{Free-space cavity optomechanics in a cryogenic environment}

A. G. Kuhn, J. Teissier, L. Neuhaus, S. Zerkani, E. van Brackel, S. Deléglise, T. Briant, P.-F. Cohadon, A. Heidmann, C. Michel, L. Pinard, V. Dolique, R. Flaminio, R. Taïbi, C. Chartier, and O. Le Traon

Citation: Applied Physics Letters 104, 044102 (2014); doi: 10.1063/1.4863666

View online: http://dx.doi.org/10.1063/1.4863666

View Table of Contents: http://scitation.aip.org/content/aip/journal/apl/104/4?ver=pdfcov

Published by the AIP Publishing

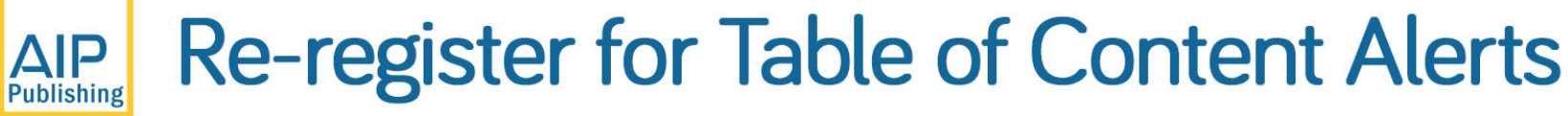




\title{
Free-space cavity optomechanics in a cryogenic environment
}

\author{
A. G. Kuhn, ${ }^{1}$ J. Teissier, ${ }^{1}$ L. Neuhaus, ${ }^{1}$ S. Zerkani, ${ }^{1}$ E. van Brackel, ${ }^{1}$ S. Deléglise,${ }^{1}$ \\ T. Briant, ${ }^{1}$ P.-F. Cohadon, ${ }^{1}$ A. Heidmann, ${ }^{1}$ C. Michel, ${ }^{2}$ L. Pinard,${ }^{2}$ V. Dolique,${ }^{2}$ R. Flaminio, ${ }^{2}$ \\ R. Taïbi, ${ }^{3}$ C. Chartier, ${ }^{3}$ and O. Le Traon ${ }^{3}$ \\ ${ }^{1}$ Laboratoire Kastler Brossel, ENS, UPMC, CNRS, Paris, France \\ ${ }^{2}$ Laboratoire des Matériaux Avancés, CNRS, IN2P3, Villeurbanne, France \\ ${ }^{3}$ ONERA, Physics and Instrumentation Department, Châtillon, France
}

(Received 6 December 2013; accepted 14 January 2014; published online 30 January 2014)

\begin{abstract}
We present a free-space optomechanical system operating in the 1-K range. The device is made of a high mechanical quality factor micropillar with a high-reflectivity optical coating atop, combined with an ultra-small radius-of-curvature coupling mirror to form a high-finesse Fabry-Perot cavity embedded in a dilution refrigerator. The cavity environment as well as the cryostat have been designed to ensure low vibrations and to preserve micron-level alignment from room temperature down to $100 \mathrm{mK}$. (C) 2014 AIP Publishing LLC. [http://dx.doi.org/10.1063/1.4863666]
\end{abstract}

Quantum optomechanics is an emerging research field, with the aim to control the quantum state of a mechanical resonator by coupling it to one or several modes of the light field, with potential applications in quantum information. So far, cooling experiments down to the quantum ground state (QGS) were conducted on resonators with masses in the ng scale or lower, and frequencies above $10 \mathrm{MHz} .{ }^{1-4}$ Reaching the quantum regime of larger mass resonators would allow to further test fundamental issues in quantum physics, such as Planck-scale physics. ${ }^{5}$

Cooling a resonator (mass $M$, angular resonance frequency $\Omega_{\mathrm{m}}$, mechanical quality factor $Q$ ) to the QGS is increasingly demanding with larger mass and lower frequency resonators. For a $4-\mathrm{MHz}$ resonator, the number $n_{T}$ $=k_{\mathrm{B}} T / \hbar \Omega_{\mathrm{m}}$ of thermal phonons is higher than 500 even in a dilution fridge at $100 \mathrm{mK}$. Hence, if a $\mathrm{GHz}$ resonator was successfully cooled to the QGS using sheer cryogenic techniques, ${ }^{1}$ lower frequency resonators require radiationpressure $^{2-4,6}$ or feedback cooling ${ }^{7,8}$ techniques. Cryogenic cooling however remains an important first step, though free-space optomechanics experiments are currently limited to temperatures in the vicinity of $10 \mathrm{~K},{ }^{9,10}$ far from the base temperature below $100 \mathrm{mK}$ routinely observed in microwave experiments. $^{1,2}$

We present here an optomechanics experiment that addresses the challenge of embedding a high- $Q \mu \mathrm{g}$-scale mechanical resonator and a high-finesse free-space cavity inside a dilution fridge environment. This experiment therefore is a free-space cryogenic implementation of cavity optomechanics, by contrast with most previous experiments which were performed either with microwave circuits, ${ }^{2}$ waveguides, ${ }^{3}$ or fiber optics. ${ }^{4}$ The microresonator is a $1-\mathrm{mm}$ long and $240-\mu \mathrm{m}$ wide quartz pillar, with a triangular section $^{11}$ (Fig. 1(a)). The effective resonator under study in the following is the fundamental compression-elongation mode of the pillar, with an expected resonance frequency close to $3.6 \mathrm{MHz}$. A huge effort has been carried out to design a resonator with very low mechanical losses. The pillar is etched out of high-purity monocrystalline quartz to take advantage of its high intrinsic $Q$, especially at low temperature, and an original concept has been proposed in order to evade the Poisson effect: ${ }^{12}$ the central pillar is linked to an outer frame by a thin membrane, monolithically micromachined on a quartz wafer. Q factors exceeding $10^{6}$ have been observed with such devices. ${ }^{11}$ As the upper side of the resonator is strain-free, it can be coated with a low-loss dielectric mirror (15 doublets of $\mathrm{SiO}_{2} / \mathrm{Ta}_{2} \mathrm{O}_{5}, 40$-ppm typical transmission and 20-ppm losses at $1064 \mathrm{~nm}$ ) without altering the $Q$, despite the poor mechanical quality of the coating layers. Roughness is on the order of $3 \AA \mathrm{rms}$ (see Fig. 1(c)). ${ }^{13}$ The resonator can be used as the end mirror of a free-space single-ended optical cavity. The first complete fabrication process and measurements were performed with a modest Q-factor of 7000, unaltered by the coating procedure.
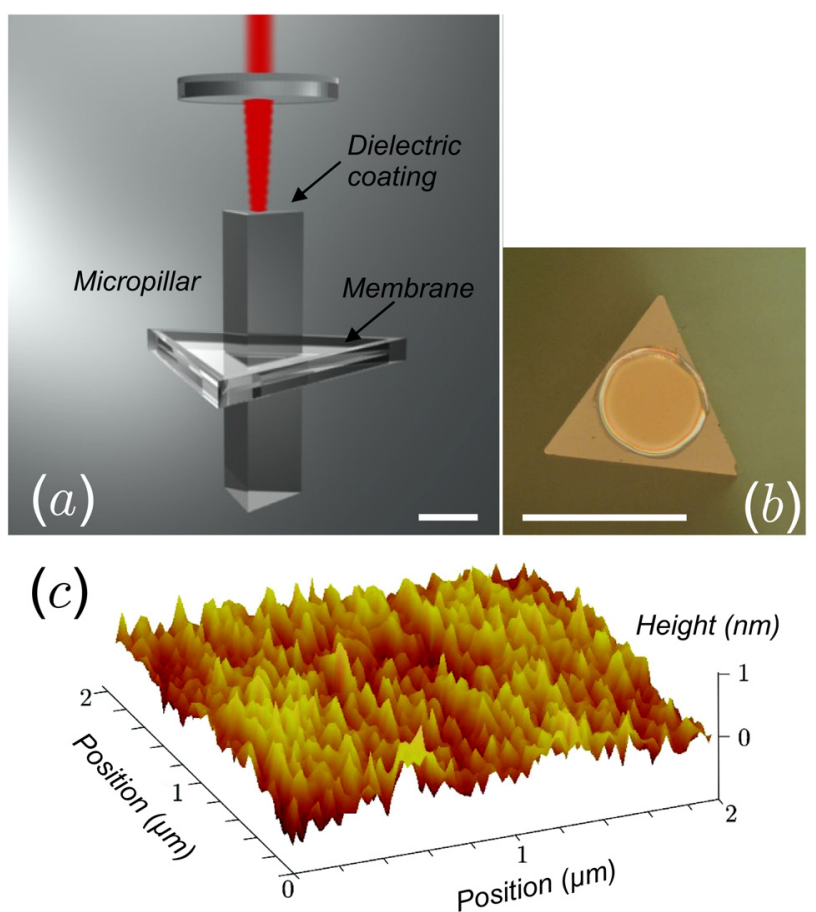

FIG. 1. Optomechanical resonator. (a): 3D view of the resonator concept. (b): Optical view of the mirror coated on top of the triangular pillar. Each white marker approximately corresponds to $200 \mu \mathrm{m}$. (c): $2 \mathrm{D}$ map of the roughness of the resonator mirror. 
Because of the maximum 100- $\mu \mathrm{m}$ diameter of the coating (see Fig. 1(b)) cavity with an optical waist $w_{0} \simeq 10 \mu \mathrm{m}$ has to be realized to avoid optical clipping losses. In planoconcave cavities, $w_{0}$ is given by $w_{0}^{2}=\frac{\lambda}{\pi} \sqrt{L(R-L)}$, where $L$ is the cavity length, $R$ is the radius of curvature (RoC) of the input mirror, and $\lambda$ is the wavelength. In order to reduce exposure to vibrations and laser frequency noise, we have designed and implemented a short, compact and thus very stable cavity, using a coupling mirror with a millimetric RoC. As such mirrors are not commercially available, we have fabricated them by $\mathrm{CO}_{2}$ laser photoablation ${ }^{14}$ on a 1-mm thick fused silica plate (Herasil from HTM-Heraeus). We have used 100-ms pulses with powers ranging from 5 to $10 \mathrm{~W}$ to obtain the targeted concave shape as surface evaporation is dominant with these parameters, and to smooth the surface on a short scale as melting is restricted to a thin layer. After fabrication, the substrate is cleaned and characterized in a class 100 clean-room environment. 2D mapping of the substrate profile obtained by profilometer measurements is shown on Fig. 2. The measured profile approximately replicates the intensity gaussian profile of the $\mathrm{CO}_{2}$ laser beam, with typical values in the $100 \mu \mathrm{m}-1 \mathrm{~mm}$ range. The local RoC has been found homogenous at the $10 \%$ level over a spot larger than $40 \mu \mathrm{m}$ around the mirror center. Astigmatism is also below $10 \%$ over the optical waist scale. High-resolution atomic force microscopy roughness measurements on a $(100-\mu \mathrm{m})^{2}$ area at the center of the mirror give typical values of $3 \AA$ rms, a value similar to the one obtained on top of the micropillar. This is confirmed again with Micromap interferometry. The concave substrate is then coated with a similar high-reflectivity dielectric mirror. The shape of the mirror is found unaltered by the coating process, replicating the initial concave profile.

The micro-pillar and the coupling mirror are both embedded inside a short copper cavity, tunable over a full free spectral range via piezo-electric actuation. A compact unit includes the Fabry-Perot cavity as well as two focussing lenses, fully translatable relatively to the mechanical resonator. The overall optical alignment is performed at room temperature and, once tightened, robust against thermal contraction between $300 \mathrm{~K}$ and $100 \mathrm{mK}$. The cavity is set up inside a dilution refrigerator especially designed for optomechanics applications. It is a horizontal model, developed in

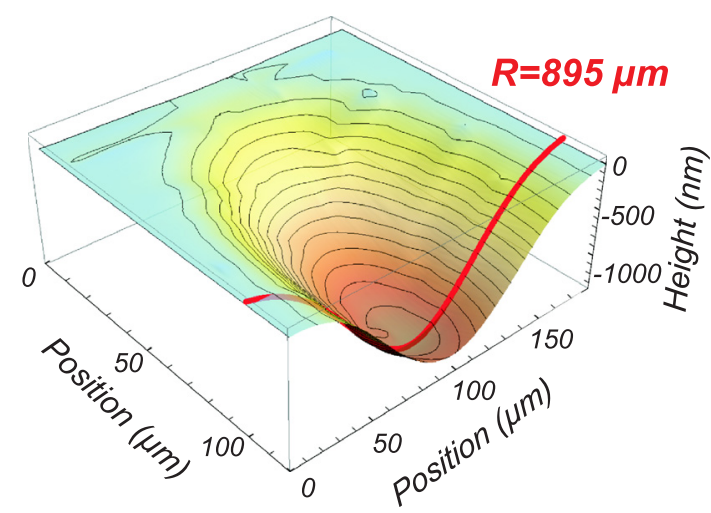

FIG. 2. Profilometer image of the concave coupling mirror, with the corresponding 1D gaussian fit, yielding a radius of curvature at the center $R=895 \mu \mathrm{m}$. collaboration with the Cryoconcept company (Fig. 3) and optimized to limit vibrations both longitudinal (which degrade the laser/cavity tuning) and transverse (which degrade the mode matching). 5 different shields (at $300 \mathrm{~K}, 65 \mathrm{~K}$, $15 \mathrm{~K}, 4 \mathrm{~K}$, and $1 \mathrm{~K}$ ) provide an efficient insulation of the cold chamber from the room temperature environment. The input laser beam can easily be injected into the cavity through a viewport (with 4 optical windows clamped to the shields along the optical path). An additional viewport, which allows for a clear view over the back of the cavity, is available on the side of the cryostat and used for alignment purposes. To accommodate the $50-\mathrm{cm}$ distance between the cavity and the $300 \mathrm{~K}$ shield, focussing optics was set up inside the cold chamber. The minimum base temperature is $30 \mathrm{mK}$ with open viewports. Cooling power is as high as $200 \mu \mathrm{W}$ at $100 \mathrm{mK}$, allowing the use of more than $1 \mathrm{~mW}$ of input laser power.

We have mounted cavities with length between 100 and $600 \mu \mathrm{m}$, and optical waists ranging from a few to $20 \mu \mathrm{m}$. The waist is measured by direct CCD-monitoring of the shape of the beam for different positions downstream the optical axis. The mode matching of the laser to the $\mathrm{TEM}_{00}$ cavity mode is about $80 \%$. The optical finesse depends on the actual waist location on the flat resonator mirror, with measured values up to 41000 at $1064 \mathrm{~nm}$, and a corresponding cavity bandwidth of $5.5 \mathrm{MHz}$ for a $340-\mu \mathrm{m}$ length. Thermal bistability is clearly visible for incident powers above $1 \mathrm{~mW}$. The absolute vibrations at the cavity location are measured at the level of $100 \mathrm{~nm} \mathrm{rms}$, and differential vibrations inside the cavity below $10 \mathrm{pm}$ rms, which can be handled by the cavity/laser tuning feedback system. All optical properties are first measured at room temperature and found preserved at low temperature.

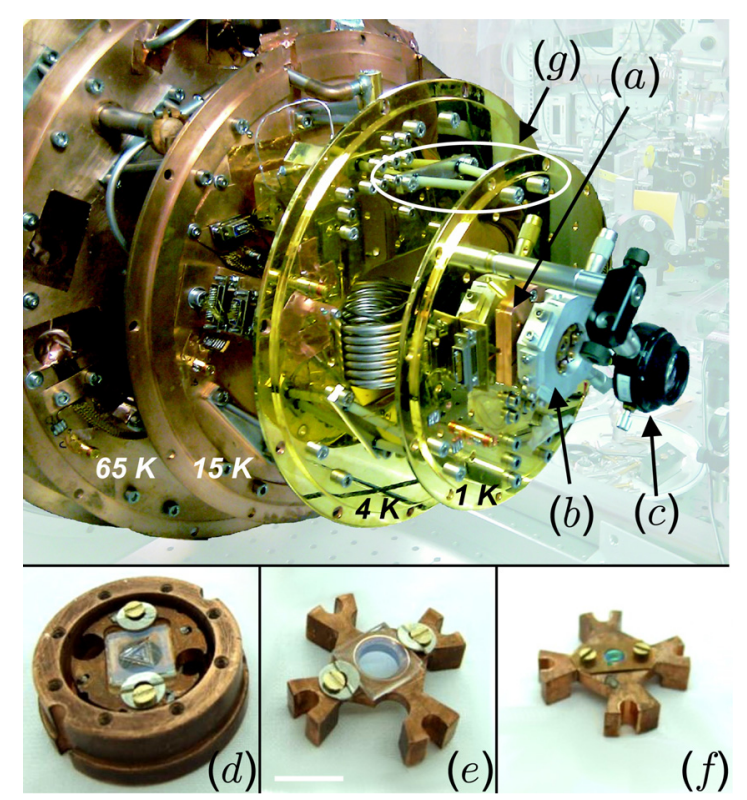

FIG. 3. Cryogenic implementation of the optomechanical resonator. The cold chamber is insulated from the room temperature environment by 5 successive shields. (a): Cavity assembly with micropillar mount (d) and coupling mirror (e), (b): removable alignment tool and first focussing lens (f), (c): second focussing lens. The white marker corresponds to $1 \mathrm{~cm}$ for all three bottom pictures. (g): Triangular pillar used to connect the different stages and limit the vibration level of the cold chamber. 


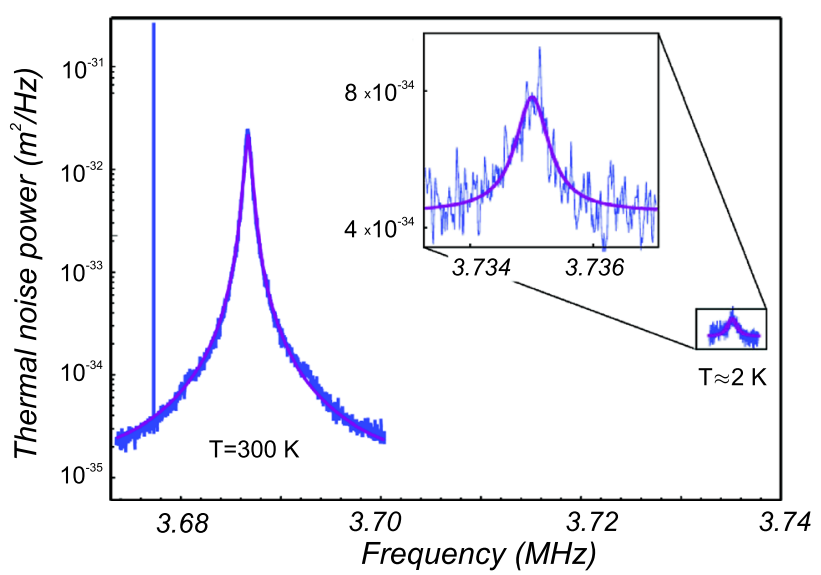

FIG. 4. Thermal noise spectrum of the resonator at room temperature $\left(P^{\mathrm{in}}=1 \mathrm{~mW}\right)$ and for a cryostat base temperature of $100 \mathrm{mK}\left(P^{\text {in }}=40 \mu \mathrm{W}\right)$. The lorentzian fit (red, see inset) allows to extract a temperature of $2 \mathrm{~K}$ for the fundamental vibration mode.

By measuring the phase noise of the reflected beam with a homodyne detection, we infer the thermal noise of the micropillar. Figure 4 shows the spectra obtained at room and cryogenic temperatures. The Lorentzian fit gives access to the mechanical characteristics of the resonator, which appear slightly dependent on the temperature: $\Omega_{\mathrm{m}} / 2 \pi \simeq 3.7 \mathrm{MHz}$, $M \simeq 100 \mu \mathrm{g}$, and $Q \simeq 7,000$. It also yields the resonator temperature. For the lowest cryostat temperature, the micro pillar is eventually thermalized at a temperature $T \simeq 2 \mathrm{~K}$ for an optical input power $P^{\mathrm{in}}=40 \mu \mathrm{W}$, because of the weak thermal link between the micropillar and the cold plate at a temperature of $100 \mathrm{mK}$, which prevents an efficient dissipation of the heat produced by intracavity light absorption and scattering. To circumvent this, the cavity will be embedded in a ${ }^{3} \mathrm{He}$ buffer gas chamber. We expect to reach a pillar temperature close to the gas temperature of $500 \mathrm{mK}$ with $1 \mathrm{~mW}$ of input power and pressures up to 1 millibar, pressure for which we have experimentally checked that a $\mathrm{Q} \simeq 10^{6}$ is not altered.

A value $C \simeq 4$ has been achieved for the optomechanical cooperativity ${ }^{15} C=8 \mathcal{F} P Q / \lambda c M \Omega_{\mathrm{m}}^{2}$ ( $P$ is the intracavity power) with $P^{\text {in }}=1 \mathrm{~mW}$. In spite of the low $\mathrm{Q}$ used here, this already corresponds to a displacement sensitivity at the standard quantum limit level at the mechanical resonance frequency. Once the system is operated in the resolved sideband regime, using a resonator with a $Q$-factor in the $10^{6}$ range (all things being equal) will allow to reach a cooperativity value in the $10^{3}$ range, and a final thermal occupation number $n_{\mathrm{f}}=n_{T} / C$ below unity.
We have developed a small-waist high-finesse FabryPerot cavity suitable for optomechanics experiments in a sub-Kelvin environment. Preliminary measurements have shown that this system is already sensitive enough to probe quantum position fluctuations of the resonator. Embedding an ultra-high $Q$ resonator in that cavity should lead to the demonstration of its QGS, opening the way to the quantum control of mechanical motion of $\mu \mathrm{g}$-scale mechanical resonators.

The authors acknowledge help from G. Vuibert for the homodyne detection setup, J. Hare for the $\mathrm{CO}_{2}$ laser setup support, M. Rosticher for advice on profilometer and AFM measurements, and C. Manquest, P. Filloux and S. Suffit at the Université Paris Diderot cleanroom. They also acknowledge financial support of the "Agence Nationale de la Recherche" program “ANR-2011-B504-028-01 MiNOToRe," by the FP7 Specific Targeted Research Project Minos and by the Marie Curie Initial Training Network $c Q O M$.

${ }^{1}$ A. D. O'Connell, M. Hofheinz, M. Ansmann, R. C. Bialczak, M. Lenander, E. Lucero, M. Neeley, D. Sank, H. Wang, M. Weides et al., Nature 464, 697 (2010).

${ }^{2}$ J. D. Teufel, T. Donner, D. Li, J. W. Harlow, M. S. Allman, K. Cicak, A. J. Sirois, J. D. Whittaker, K. W. Lehnert, and R. W. Simmonds, Nature 475, 359 (2011).

${ }^{3}$ J. Chan, T. P. Mayer Alegre, A. H. Safavi-Naeini, J. T. Hill, A. Krause, S. Groblacher, M. Aspelmeyer, and O. Painter, Nature 478, 89 (2011).

${ }^{4}$ E. Verhagen, S. Deléglise, S. Weis, A. Schliesser, and T. J. Kippenberg, Nature 482, 63 (2012).

${ }^{5}$ F. Marin, F. Marino, M. Bonaldi, M. Cerdonio, L. Conti, P. Falferi, R. Mezzena, A. Ortolan, G. A. Prodi, L. Taffarello et al., Nat. Phys. 9, 71 (2012).

${ }^{6}$ O. Arcizet, P.-F. Cohadon, T. Briant, M. Pinard, and A. Heidmann, Nature 444, 71 (2006).

${ }^{7}$ P.-F. Cohadon, M. Pinard, and A. Heidmann, Phys. Rev. Lett. 83, 3174 (1999).

${ }^{8}$ O. Arcizet, P.-F. Cohadon, T. Briant, M. Pinard, A. Heidmann, J.-M. Mackowski, C. Michel, L. Pinard, O. Français, and L. Rousseau, Phys. Rev. Lett. 97, 133601 (2006).

${ }^{9}$ E. Serra, A. Borrielli, F. S. Cataliotti, F. Marin, F. Marino, A. Pontin, G. A. Prodi, and M. Bonaldi, Phys. Rev. A 86, 051801(R) (2012).

${ }^{10}$ T. P. Purdy, R. W. Peterson, and C. A. Regal, Science 339, 801 (2013).

${ }^{11}$ A. G. Kuhn, M. Bahriz, O. Ducloux, C. Chartier, O. Le Traon, T. Briant, P.-F. Cohadon, A. Heidmann, C. Michel, L. Pinard, and R. Flaminio, Appl. Phys. Lett. 99, 121103 (2011).

${ }^{12}$ M. Bahriz and O. Le Traon, French patent 10/02829 (July 2010).

${ }^{13}$ R. Flaminio, J. Franc, C. Michel, N. Morgado, L. Pinard, and B. Sassolas, Classical Quantum Gravity 27, 084030 (2010).

${ }^{14}$ D. Hunger, C. Deutsch, R. J. Barbour, R. J. Warburton, and J. Reichel, AIP Adv. 2, 012119 (2012).

${ }^{15}$ S. Weis, R. Rivière, S. Deléglise, E. Gavartin, O. Arcizet, A. Schliesser, and T. J. Kippenberg, Science 330, 1520 (2010). 